OPEN ACCESS

Edited by:

Valentina Stefanetti,

University of Perugia, Italy

Reviewed by:

Denis V. Kolbasov,

Federal Research Center of Virology and Microbiology, Russia Melina Fischer,

Friedrich-Loeffler-Institute, Germany

${ }^{*}$ Correspondence: Aiping Wang pingaw@126.com

Specialty section: This article was submitted to Veterinary Infectious Diseases, a section of the journal Frontiers in Veterinary Science

Received: 27 October 2020 Accepted: 11 December 2020 Published: 05 February 2021

Citation:

Jia R, Zhang G, Liu H, Chen Y, Zhou J, Liu Y, Ding P, Wang Y, Zang W and Wang A (2021) Novel Application of Nanofluidic Chip Digital PCR for Detection of African Swine Fever Virus. Front. Vet. Sci. 7:621840 doi: 10.3389/fvets.2020.621840

\section{Novel Application of Nanofluidic Chip Digital PCR for Detection of African Swine Fever Virus}

\author{
Rui Jia ${ }^{1}$, Gaiping Zhang ${ }^{1}$, Hongliang Liu ${ }^{2}$, Yumei Chen ${ }^{1,2}$, Jingming Zhou ${ }^{1}$, Yankai Liu ${ }^{2}$, \\ Peiyang Ding ${ }^{1}$, Yanwei Wang ${ }^{2}$, Weimin Zang $^{2}$ and Aiping Wang ${ }^{1 *}$ \\ ${ }^{1}$ School of Life Sciences, Zhengzhou University, Zhengzhou, China, ${ }^{2}$ Henan Zhongze Biological Engineering Co. LTD, \\ Zhengzhou, China
}

African swine fever virus (ASFV) gives rise to a grievous transboundary and infectious disease, African swine fever (ASF), which has caused a great economic loss in the swine industry. To prevent and control ASF, once suspicious symptoms have presented, the movement of animal and pork products should be stopped, and then, laboratory testing should be adopted to diagnose ASF. A method for ASFV DNA quantification is presented in this research, which utilizes the next-generation PCR platform, nanofluidic chip digital PCR (cdPCR). The cdPCR detection showed good linearity and repeatability. The limit of detection for cdPCR is 30.1995 copies per reaction, whereas no non-specific amplification curve was found with other swine viruses. In the detection of 69 clinical samples, the cdPCR showed significant consistency [91.30\% (63/69)] to the Office International des Epizooties-approved quantitative PCR. Compared with the commercial quantitative PCR kit, the sensitivity of the cdPCR assay was $86.27 \%(44 / 50)$, and the specificity was $94.44 \%$ (17/18). The positive coincidence rate of the cdPCR assay was 88\% (44/50). The total coincidence rate of the cdPCR and kit was 89.86\% (62/69), and the kappa value reached $0.800(P<0.0001)$. This is the first time that cdPCR has been applied to detecting ASFV successfully.

Keywords: African swine fever virus, chip digital PCR, sensitive detection, application, nanofluidic

\section{INTRODUCTION}

African swine fever (ASF) was first reported in Kenya, Eastern Africa, in 1921 and then gradually swept across the globe to this day $(1,2)$. With the death rate of the swine approaching $100 \%$, ASF is putting downward pressure on the global economy and is a disaster for the pig industry $(3,4)$. African swine fever virus (ASFV), the pathogen causing ASF, is a large double-stranded DNA virus with an envelope and is the only member of the Asfivirus genus in the Asfarviridae family (5). The genome size of ASFV is from 170 to $190 \mathrm{~kb}$, so it belongs to the nucleocytoplasmic large DNA viruses $(6,7)$.

Domestic pigs with ASFV infections have serious clinical manifestations such as acute hemorrhagic fever, dyspnea, serous or mucopurulent conjunctivitis, bloody dysentery, vomiting, among others (8). Currently, there are no effective treatments, and vaccine research is progressing slowly. Once suspicious symptoms of ASF presented, the most valid measurements are to firstly stop all circulation of animals and pork products, for example, via animal isolation and traffic restriction (9-11). ASF can then be confirmed by a laboratory test (12). 
The laboratory diagnostic approaches of ASF are mainly divided into two groups: one includes isolating the virus, detecting virus antigens and genomic DNA, whereas the other aims at detecting an antibody $(12,13)$. Polymerase chain reaction (PCR) technique is the most mature molecular method for determining virus genomic DNA/RNA. Conventional PCR (14) and fluorescent quantitative PCR (qPCR) (15) have been applied in testing ASFV. Several PCR technologies have been established to achieve quantitative analysis for the concentration of virus DNA during amplification. Real-time fluorogenic qPCR is the most frequently used form of qPCR, in which concentrations of samples are calculated from initial concentrations of standard sample templates. Currently, although qPCR has been used in ASFV detection studies to measure the virus genomic DNA (1621), digital PCR is getting increasingly popular because it realizes absolute quantification without reliance on external standards, standard curves, and the cycle within the amplification process that the reporter dye signal exceeds a threshold [cycle threshold (CT) value] (22).

Nanofluidic chip digital PCR (cdPCR), a type of digital PCR supported by QuantStudio 3D (Applied Biosystems, US), adopts a sealed chip that partitions samples into thousands of reaction wells to run independent PCR amplifications. When amplifications are finished, the concentration of the target gene in the original sample is calculated by counting and converting positive wells, which have positive amplification of the viral target gene using the Poisson model correction coefficient $(22,23)$. Another superiority of cdPCR is the high sensitivity, which makes it a dream platform for studying $(24,25)$ low-level pathogen detection $(26,27)$ as well as absolute quantification of viral load (28).

This study focuses on the application of cdPCR, in which ASFV is detected by designing a pair of primers and the minor groove binder (MGB) probe in the portion sequence of the ASFV B646L gene. Applicability of this new ASFV diagnosis methods is evaluated in terms of sensitivity, specificity, and coincidence rate with qPCR approved by the Office International des Epizooties (OIE) and commercial qPCR kits.

\section{MATERIALS AND METHODS}

\section{Probe and Primers}

We designed a set of the MGB probe [5'-(FAM)ACTGGGACAACCAAAC-3' -(MGB)], upstream primer (ASFVFor: $5^{\prime}$-ACGTTTCCTCGCAACGGATA-3') and downstream primer (ASFV-Back: 5'-CGTGTAAACGGCGCCCTCTAA-3'), which aimed at the B646L gene (Genebank: MK128995.1) using PRIMER EXPRESS software (version 1.5, Applied Biosystems, USA). The size of the target gene was approximately $63 \mathrm{bp}$. Primers and the probe sequences were compared with genes of some various ASFV strains sequences in the GenBank database (Table 1).

\section{Construction of Standard Plasmid}

A 1,941 bp complete fragment of ASFV B646L geneencoded p72 protein and the ASFV B646L gene with EcoRI/XbaI restriction enzyme cutting site were obtained from the pUC57-p72 plasmid (synthesized by Sangon, Shanghai) by PCR using primers p72-Fwd: 5' CGGAATTCATGGCATCAGGAGGAGC-3' and p72-Rev: 5'-GCTCTAGATTAATGATGATGATGATGATGGGTACTG TAACG-3'. Then, the B646L gene was recombined with pFastBacI vector (Promega, USA) and transformed into DH5 $\alpha$ (Takara, Dalian, China). The recombinant plasmid, pFastBacIp72 plasmid, was extracted using Omega Plasmid mini kit (Omega, US). Restriction enzyme digestion and sequencing were used to determine whether the target fragment could be inserted correctly.

\section{Samples Preparation}

The protocols of standard templates and clinical sample preparations were as follows. The concentration of the standard plasmid constructed in Section Construction of Standard Plasmid was detected using the NanoDrop One (ThermoFisher, US, AZY1812131) and diluted to the appropriate copy number, which began with $10^{10}$ copies/ml to 10 -fold dilution. Copy number calculating formulas was shown as below.

$$
\begin{aligned}
& \text { Copy number }(\text { copies } / \mathrm{ml}) \\
& =\frac{6.02 \times 10^{23}(\text { copies } / \mathrm{mol}) \times C(\mathrm{~g} / \mathrm{ml})}{n(b p) \times\left(1.096 \times 10^{-23} \mathrm{~g} / \mathrm{bp}\right)(\mathrm{g} / \mathrm{mol})}
\end{aligned}
$$

where $\mathrm{C}(\mathrm{g} / \mathrm{ml})$ means the concentration of standard templates, and $\mathrm{n}(\mathrm{bp})$ means the genome size in base pairs.

Plasmids ranged from $10^{10}$ to $10^{0}$ copies $/ \mathrm{ml}$ were as templates and positive controls for subsequent experiments. Inactivated clinical serum samples were obtained from the Henan Animal Husbandry Bureau and pig farms in Henan province, China. ASFV genomic DNA of clinical samples was extracted from swine serum samples by DNA Extraction Kit (Takara MiniBEST Viral DNA/RNA Extraction Kit, Takara, Dalian, China).

\section{Optimal Conditions of Quantitative PCR}

An ABI 7500 Real-time PCR system (Applied Biosystems, USA) was used as a fluorescence quantification platform in this study. The reaction system was $10 \mu \mathrm{l}$, including $5 \mu \mathrm{l}$ TaqMan Universal Master Mix II with uracil-N-glycosylase (purchased from Applied Biosystems, USA), $0.4 \mu \mathrm{l}$ sense primer (ASFVFor), $0.4 \mu \mathrm{l}$ anti-sense primer (ASFV-Back), $0.4 \mu \mathrm{l}$ of probe, 1.8$\mu l$ nuclease-free water (Promega, USA), and $2 \mu \mathrm{l}$ of template. The optimal concentrations of primers and the probe were then measured when the ASFV pFastBacI-p72 plasmid was $1 \times 10^{8}$ copies/ml. Primers with optimal concentration were determined by $12.5,25,50$, and $100 \mu \mathrm{M}$; meanwhile, the probe with optimal concentration was selected by $1.25,2.5,5$, and $10 \mu \mathrm{M}$. The qPCR program was carried out as follows: initial denaturation at $95^{\circ} \mathrm{C}$ for $10 \mathrm{~min}$, and at $95^{\circ} \mathrm{C}$ for $15 \mathrm{~s}$, cycling 40 times, and at $60^{\circ} \mathrm{C}$ holding for $45 \mathrm{~s}$. Negative and positive controls were set at the same time in a run.

\section{Digital PCR}

QuantSudio $^{\text {TM }}$ 3D Digital PCR System (ThermoScientific, US) was used as a cdPCR amplification platform. The volume of the reaction mixture was $20 \mu \mathrm{l}$, containing $10 \mu \mathrm{l} 2 \times$ QuantSudio $^{\mathrm{TM}}$ 
TABLE 1 | The primers and MGB probe were aligned with 53 ASFV epidemic strains and 5 other swine pathogenes.

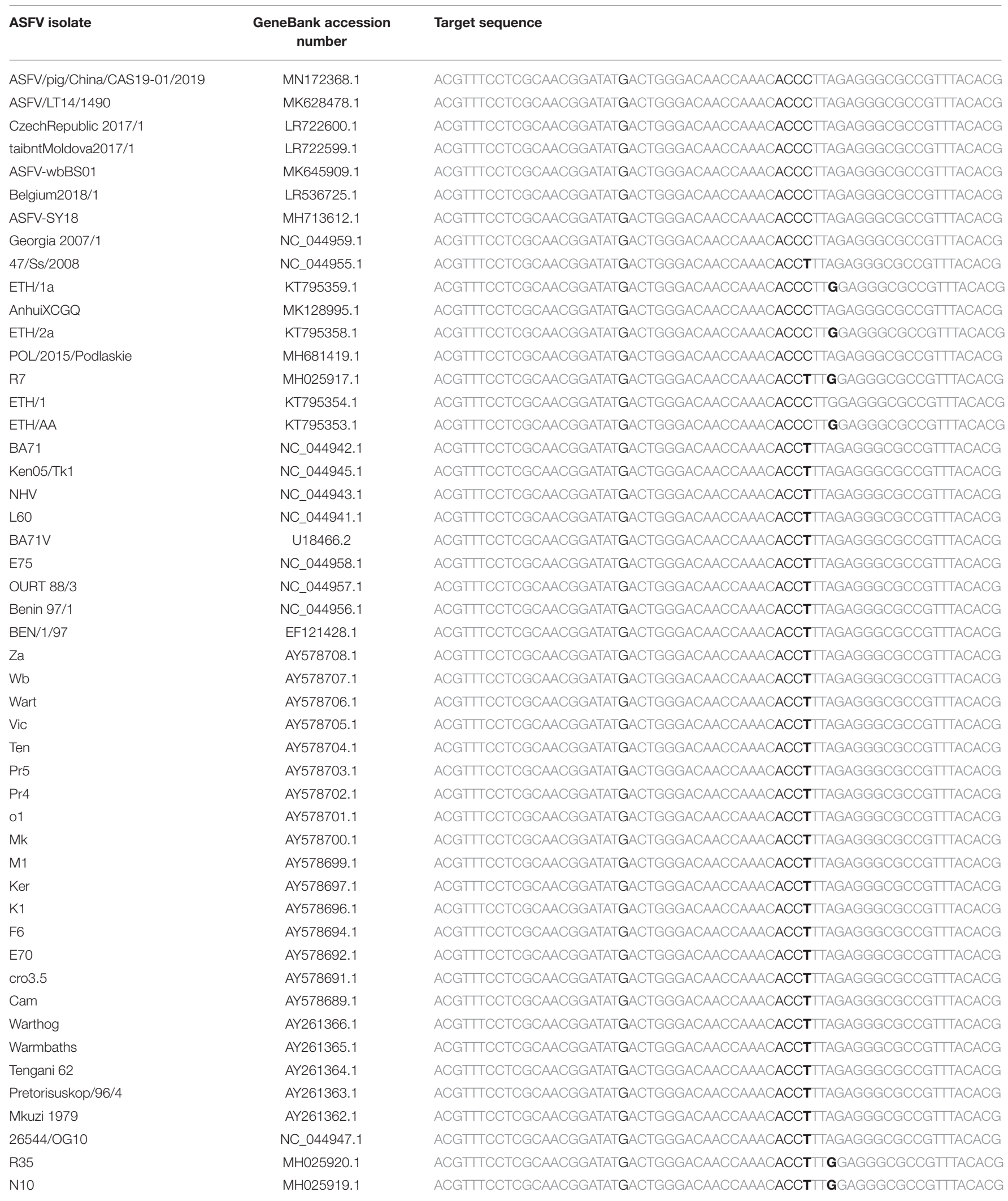


TABLE 1 | Continued

\begin{tabular}{|c|c|c|}
\hline ASFV isolate & $\begin{array}{c}\text { GeneBank accession } \\
\text { number }\end{array}$ & Target sequence \\
\hline Ken06.Bus & NC_044946.1 & ACGTाCCTCGCAACGGATATGACTGGGACAACCAAACACCT $\| \mathbf{G G A G G G C G C C G T \prod A C A C G ~}$ \\
\hline $\mathrm{Kn}$ & AY578698.1 & ACGTTCCTCGCAACGGATATGACTGGGACAACCAAACACCT $\| \mathbf{G G A G G G C G C C G T T A C A C G ~}$ \\
\hline RSA_2_2008 & MN336500.1 & ACGTTCCTCGCAACGGATATGACTGGGACAACCAAACACCTGTAGAGGGCGCCGTTACACG \\
\hline PRRSV & MH500776.1 & No matches \\
\hline PEDV & KY496315.1 & No matches \\
\hline PCV2 & MK604485 & No matches \\
\hline
\end{tabular}

The left grey sequence is sense primer, the right grey sequence is anti-sense primer, and the middle grey sequence is MGB probe. Moreover, the bold letters indicate the mutated bases.

3D Digital PCR Master Mix (v2), $1.8 \mu$ l of each primer with optimal concentration determined by qPCR, $1.8 \mu \mathrm{l}$ of the probe with optimal concentration determined by qPCR, $2.6 \mu \mathrm{l}$ nuclease-free water (ThermoScientific, US), and $2 \mu \mathrm{l}$ of DNA template. After sufficient mixed and briefly centrifuged, the 14.5 $\mu l$ cdPCR reaction mixture was immediately loaded to the chips. Negative control and positive control were set for each test. Three replicates of the standard plasmid template were performed in each run. The program was in operation at $96^{\circ} \mathrm{C}$ for $10 \mathrm{~min}$ as a predenaturation step, at $60^{\circ} \mathrm{C}$ for $2 \mathrm{~min}$, and at $98^{\circ} \mathrm{C}$ for $30 \mathrm{~s}$, cycling 39 times, and finally, at $60^{\circ} \mathrm{C}$ for $2 \mathrm{~min}$ as a final elongation step.

\section{Limit of Detection for Chip Digital PCR}

The limit of detection (LOD) for cdPCR was determined by the continuous dilution method. At the same time, the same templates were used for qPCR, approved by OIE (12) to compare the LOD between the two methods. The two amplification methods were repeated three times, and the data were analyzed statistically by logistic regression (Statistica 64, USA) (29).

\section{Specificity Analysis}

In this analysis, the classical swine fever virus strain Shimen (AF092448), the porcine circovirus 2 strain HN-LB-16 (MK604485), the porcine reproductive and respiratory syndrome virus strain NADC30 (MH500776.1), and the porcine parvovirus strain China (AY583318.1) were kindly provided by Henan Agricultural University (Zhengzhou, Henan, China), and the porcine epidemic diarrhea virus strain $\mathrm{CH} /$ hubei/2016 (KY496315.1) was kindly provided by Jilin University (Changchun, Jilin, China). All these pathogens were detected by the nanofluidic cdPCR assay as nucleic acid templates.

\section{Repeatability Evaluation}

The repeatability of cdPCR was evaluated by using the continuous dilution of ASFV standard plasmid containing $10^{\circ}$, $10^{1}, 10^{2}, 10^{3}$, and $10^{4}$ copies $/ \mathrm{ml}$ as templates. On different days, three experiments were carried out, and each template in each experiment was repeated three times. The coefficient of variation (CV) was measured to analyze repeatability.

\section{Comparison of Chip Digital PCR With Quantitative PCR Approved by Office International des Epizooties and Commercial Kits}

Comprehensive comparisons of cdPCR with qPCR approved by OIE and commercial kit VetMAX $^{\mathrm{TM}}$ African Swine Fever Virus Detection Kit, Thermofisher, US) were carried on by detecting 69 clinical samples. SPSS (version 21.0, IBM, USA) software and GraphPad Prism software (version 7.04; LA Jolla, California, USA) were used for statistical analysis, including the compliance rate, Bland and Altman analyses, and linear regression with the confidence limit of $95 \%(P<0.05)$.

\section{RESULTS}

\section{Construction of Standard Plasmid and Identification of Target Gene}

The standard plasmid, pFastBacI-p72, was successfully constructed and identified by PCR and sequencing (Figure 1). The recombination process of objective gene ASFV p72 (B646L) and vector pFastBacI is shown in Figure 1A. The target gene, ASFV B646L, was amplified by PCR with $1,941 \mathrm{bp}$ (Figure 1B) and spliced into two cleavage sites of restriction enzyme EcoRI and XbaI of vector pFastBacI. As shown in Figure 2A, double-stranded DNA sequences of the MGB probe and primers were marked in different colors within the conserved region of ASFV B646L. The size of the target gene amplified by cdPCR was $\sim 63 \mathrm{bp}$. A single band of approximately 63 bp was obtained from PCR amplification products via $1 \%$ agarose gel electrophoresis (Figure 2B).

\section{Reaction Conditions of Quantitative PCR}

The optimum reaction condition for qPCR was detected via using a series of different concentrations of primers and the probe. The optimal concentration of primers was $12.5 \mu \mathrm{M}$, and the optimal concentration of the probe was $10 \mu \mathrm{M}$, at that time the $\mathrm{C}_{\mathrm{T}}$ value was minimum (Figure 3A). The optimum reaction system and the program are shown in Figures 3B,C. 


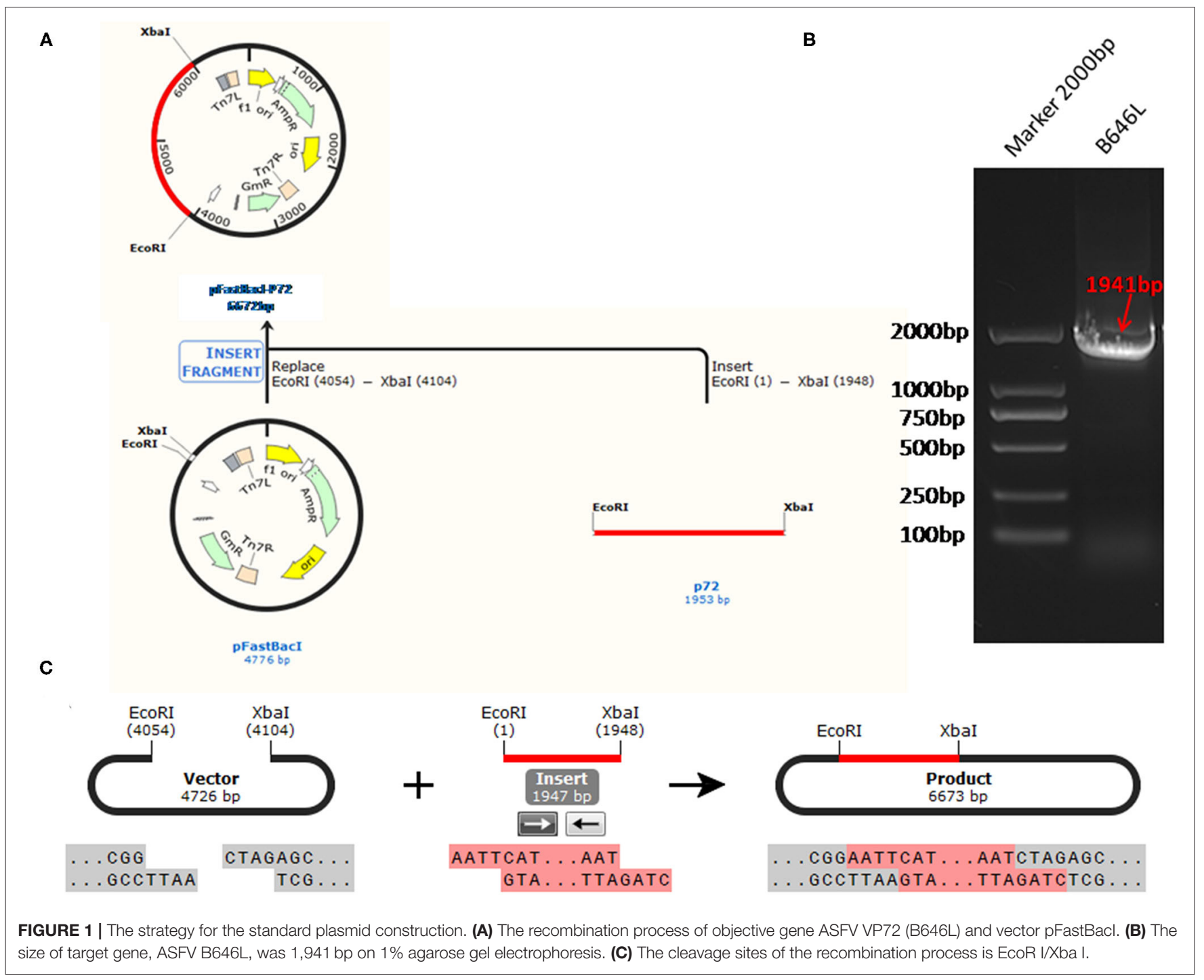

\section{Linear Standard Curve of Chip Digital PCR Assay}

Using 10-fold diluted ASFV standard plasmid of $10^{4}-10^{-1}$ copies $/ \mathrm{ml}$ as templates, the standard curve of cdPCR was established. At the same time, the standard curve of qPCR confirmed by OIE was created by the same standard plasmid of $10^{9}-10^{0}$ copies $/ \mathrm{ml}$. The trend line was highly linear with the assumed concentration for both cdPCR (Figure 4A) and qPCR (Figure 4B). The cdPCR assay proved greater linearity with an $R^{2}$ of 0.9985 than the qPCR assay with an $R^{2}$ of 0.9881 (Figure 4).

\section{Limit of Detection of Curve of Chip PCR Assay}

The LODs for both cdPCR and qPCR approved by OIE were determined using the same set of primers and the probe with ASFV standard plasmid diluted 10 times as templates. The results are shown in Figure 5. Using the least-squares modeling approach and logistic regression analysis, the $\mathrm{LOD}_{95 \%}$ of the
cdPCR assay was $1.48 \log 10$ copies per reaction, that is, 30.1995 copies per reaction (Figures $5 \mathbf{A}, \mathbf{C}$ ), and the $\mathrm{LOD}_{95 \%}$ of the $\mathrm{qPCR}$ assay was estimated as three $\log 10$ copies per reaction, that is, 1,000 copies per reaction (Figures 5B,D). Hence, the $\mathrm{LOD}_{95 \%}$ of cdPCR assay was approximately 33 times higher than that of the qPCR assay. The cdPCR assay was more sensitive than the qPCR assay.

\section{Specificity Analysis}

To analyze the specificity of cdPCR, DNA and complementary DNA, extracted from other swine viruses containing classical swine fever virus, porcine parvovirus, porcine circovirus 2, porcine reproductive and respiratory syndrome virus, and porcine epidemic diarrhea virus, were used as templates, and ASFV pFastBacI-p72 standard plasmid was used as a positive control in specificity assay. The standard plasmid was positive, but nucleic acid templates of the other five pathogens were negative (Figure 6 and Table 2). The result was strongly in line with our theorized expectations that the sequences of primers and probe for the ASFV cdPCR 


\section{A}

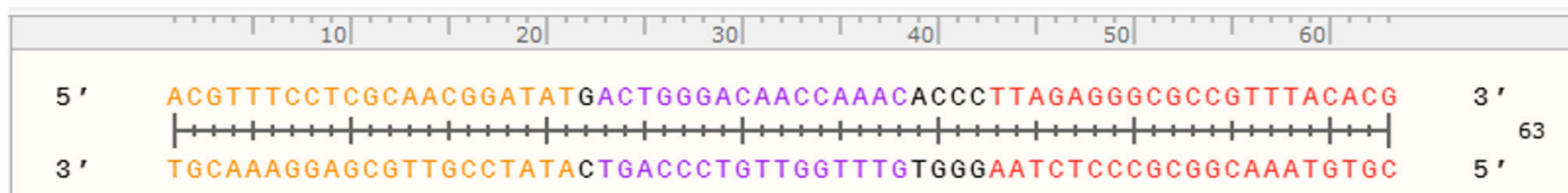

B

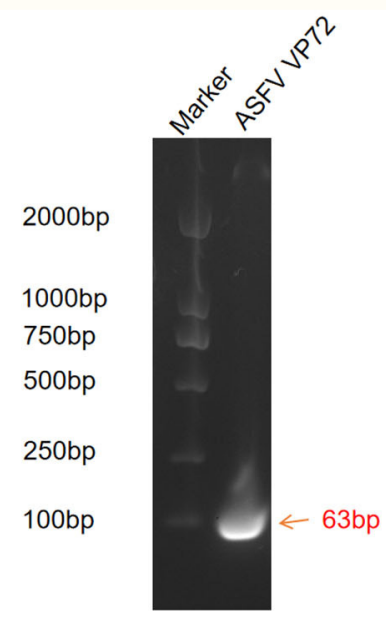

FIGURE 2 | Identification of target gene. (A) Target nucleotide sequences of MGB probe and primers for cdPCR within the conserved region of ASFV B646L gene. Forward primer was marked in orange, reverse primer was marked in red and the probe was marked in purple. (B) Amplification products were analyzed by agarose gel electrophoresis.

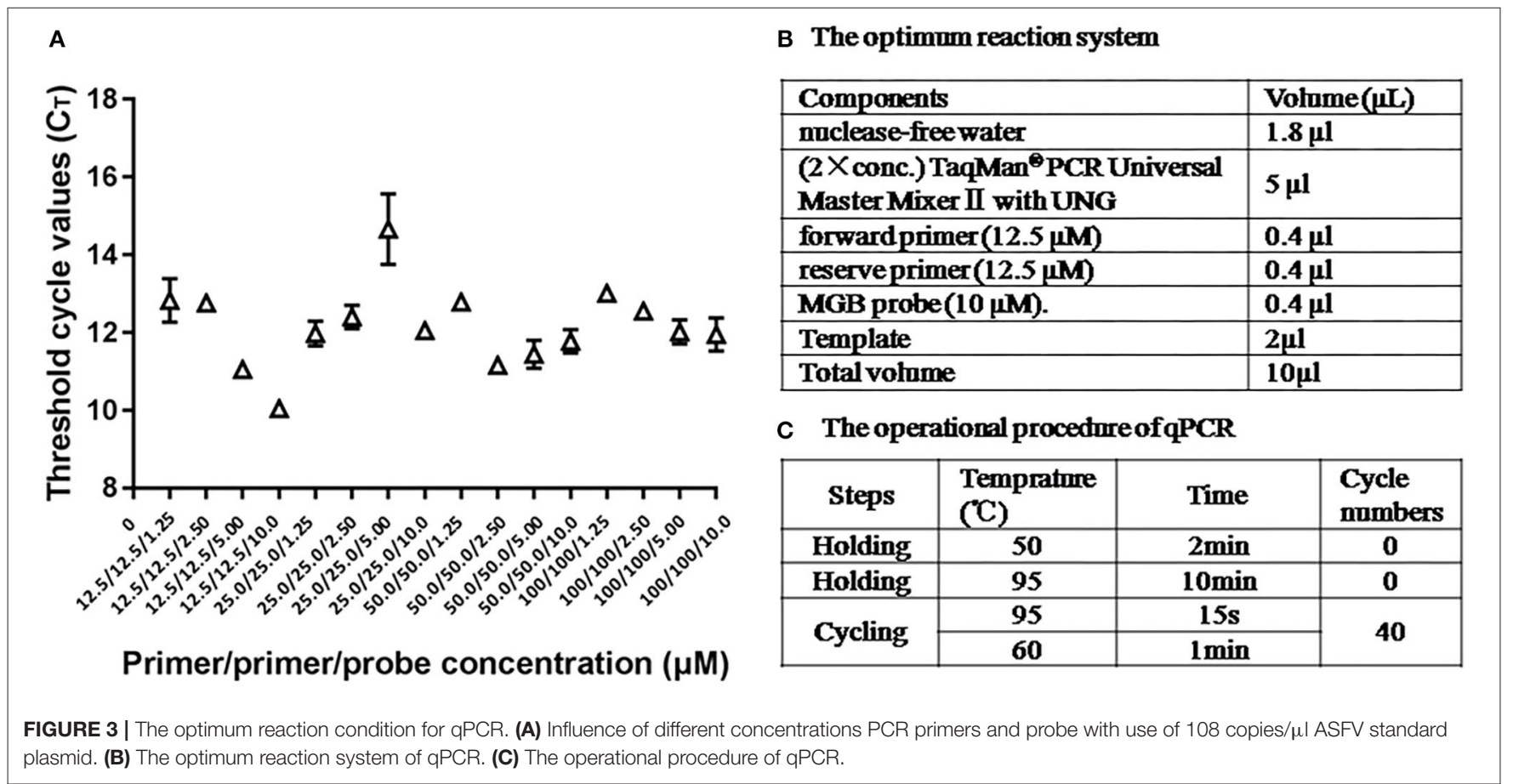

did not match with the nucleic acid sequences of any other swine pathogens (Table 1). All results mentioned earlier demonstrated that the ASFV cdPCR detection method had good specificity.

\section{Repeatability Analysis}

Using serially diluted standard plasmids as templates for cdPCR amplification, three independent experiments were performed by different operators at different times. The cdPCR assay displayed 
A

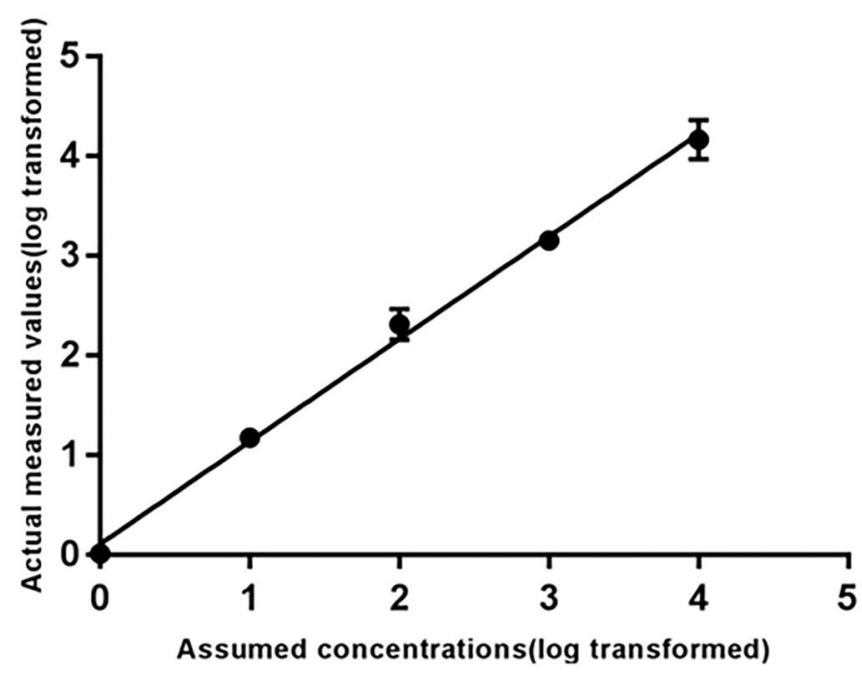

B

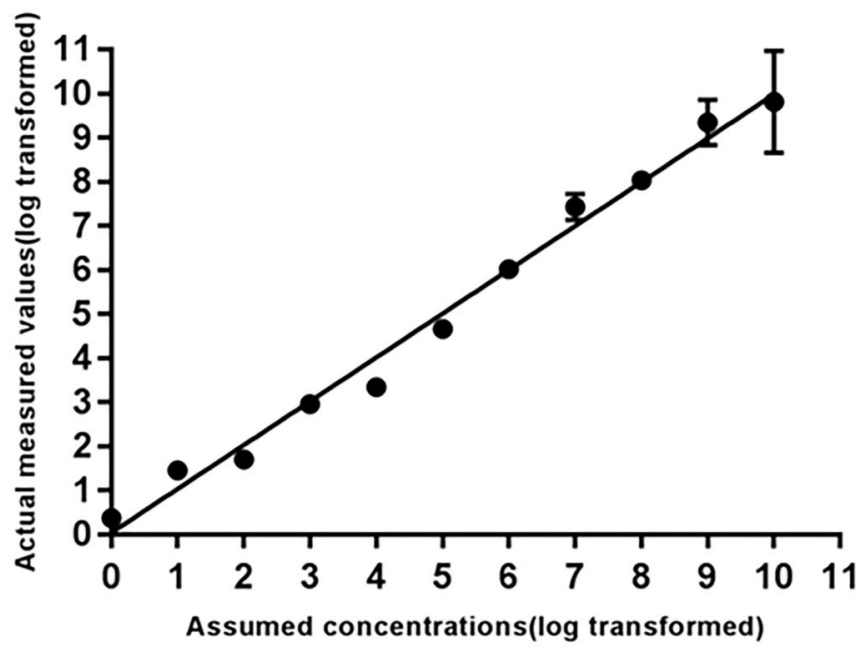

$Y=1.029 x+0.1048$

$R^{2}=0.9985$

$P<0.0001$

\section{$Y=0.9944 x+0.03865$ $R^{2}=0.9881$ $P<0.0001$}

FIGURE 4 | The standard curve of cdPCR and GPCR. (A) The standard curve of CdPCR. The slope of this linear fitting equation is 1.029 and the Y-intercept is 0.1048. (B) The standard curve of qPCR. The slope of this linear fitting equation is 0.9944 and the Y-intercept is 0.03865.

good repeatability and a low coefficient of variation between most dilution points (Figure 7). The cdPCR assay had an average CV\% of $9.56 \%$, which was lower than the average CV of $12.67 \%$ of qPCR approved by OIE, resulting in an average decrease in CV\% of $26.99 \%$ (Figure 7 ).

\section{Analysis With Clinical Samples}

To calculate the coincidence rate of the cdPCR method to detect ASFV, we compared, respectively, the cdPCR method established in this study with the qPCR approved by OIE and commercial qPCR kit (VetMAX ${ }^{\mathrm{TM}}$ African Swine Fever Virus Detection Kit, Thermofisher, US), by testing 69 swine serum samples.

As shown in Table 3A, the cdPCR and qPCR approved by OIE have, respectively, detected 50 and 48 positive samples in the clinical diagnosis of 69 domestic pigs. The sensitivity of the cdPCR assay was $95.83 \%$ (46/48), and the specificity was $94.44 \%$ $(17 / 21)$. The positive coincidence rate of the cdPCR assay was $92 \%(46 / 50)$. The total coincidence rate of the two methods was $91.30 \%(63 / 69)$, and the kappa value reached $0.789(P<$
0.0001). There was significant consistency between the two from the results. Furthermore, quantitation of the correlation between the two was analyzed by Pearson correlation and linear regression analysis on 46 positive samples (Figure 8). The quantitative analysis of the correlation between the two showed that they had a good correlation because the $R^{2}$ value of linear regression was $0.984(P<0.0001)$ (Figure 8A). The standard deviation of cdPCR was lower than that of qPCR by Mann-Whitney $U$ test (Figure 8B). Bland and Altman analyses plots (Figure 8C) demonstrated that $5.797 \%(4 / 69)$ dots were outside the region between 95\% lower limit of agreement and 95\% upper limit of agreement, and the bias value for this agreement's range was 1,381 copies/ml $(P<0.05)$ by Graphpad Prism 7.04.

The data in Table 3B show that 45 of 69 samples were judged to be positive by VetMAX ${ }^{\mathrm{TM}}$ African Swine Fever Virus Detection Kit. The sensitivity of the cdPCR assay was $86.27 \%$ (44/50), and the specificity was $94.44 \%$ (17/18). Furthermore, the positive coincidence rate and the overall coincidence rate of the cdPCR assay were $88 \%(44 / 50)$ and $89.86 \%(62 / 69)$, 
A

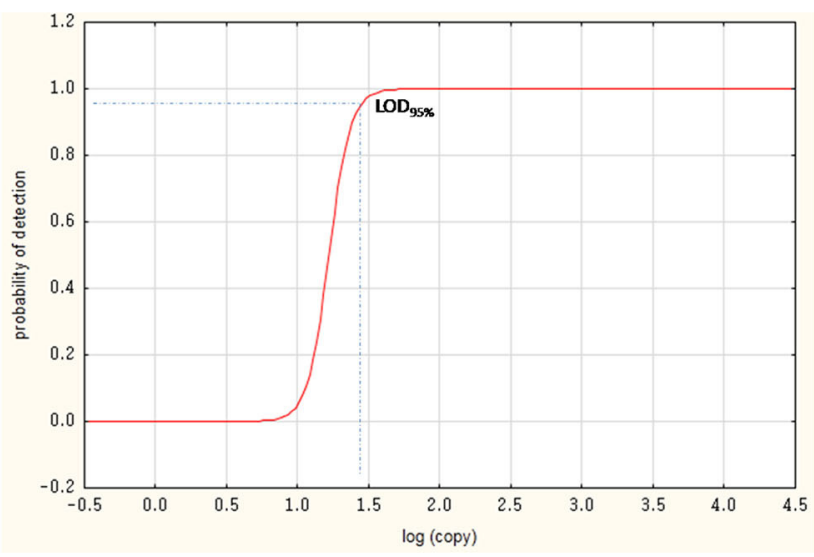

B

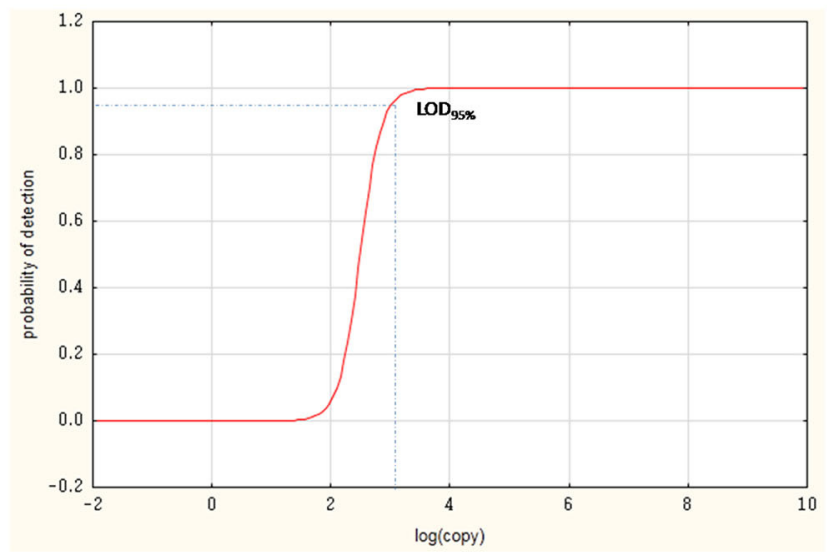

c

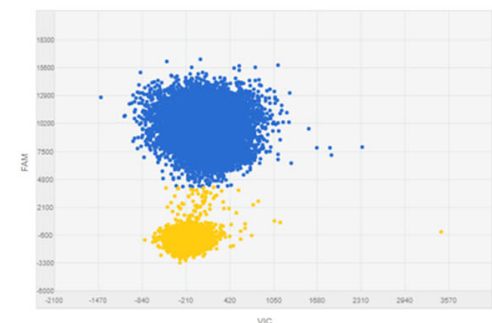

10000 copies

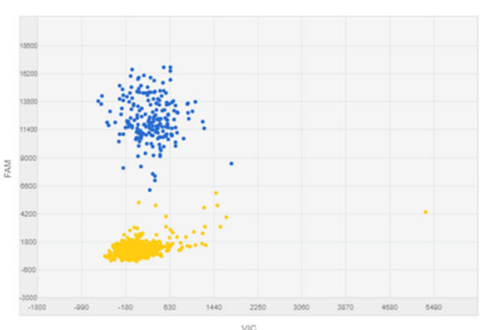

10copies

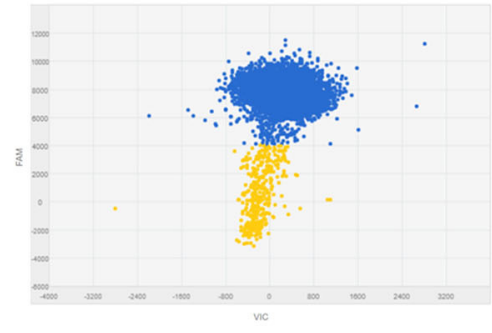

1000copies

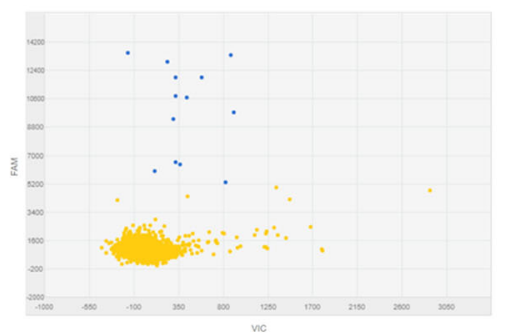

1 copies

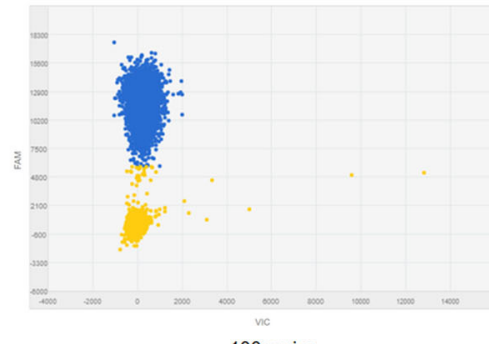

100 copies

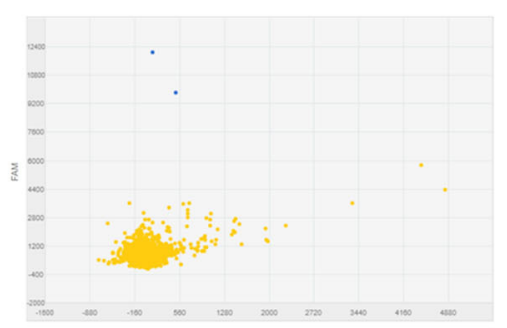

0.1 copies

D

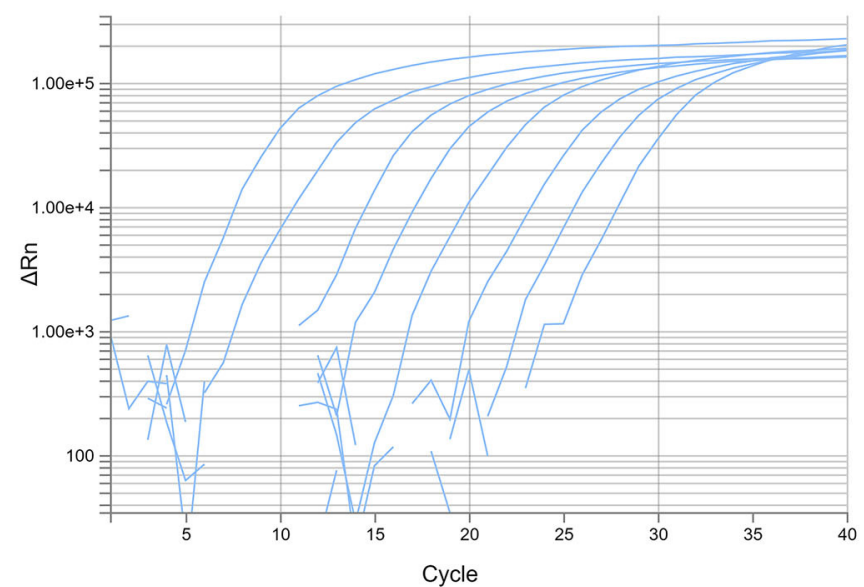

FIGURE 5 | The LOD $95 \%$ of CdPCR and APCR assay. Logit analysis plots of the cdPCR (A) and APCR (B) used in the study show the LOD $95 \%$, which are the minimum amounts of DNA detectable with a $95 \%$ probably. The amplification curve of cdPCR (C) and GPCR (D) is obtained with 10-fold diluted standard plasmids. 


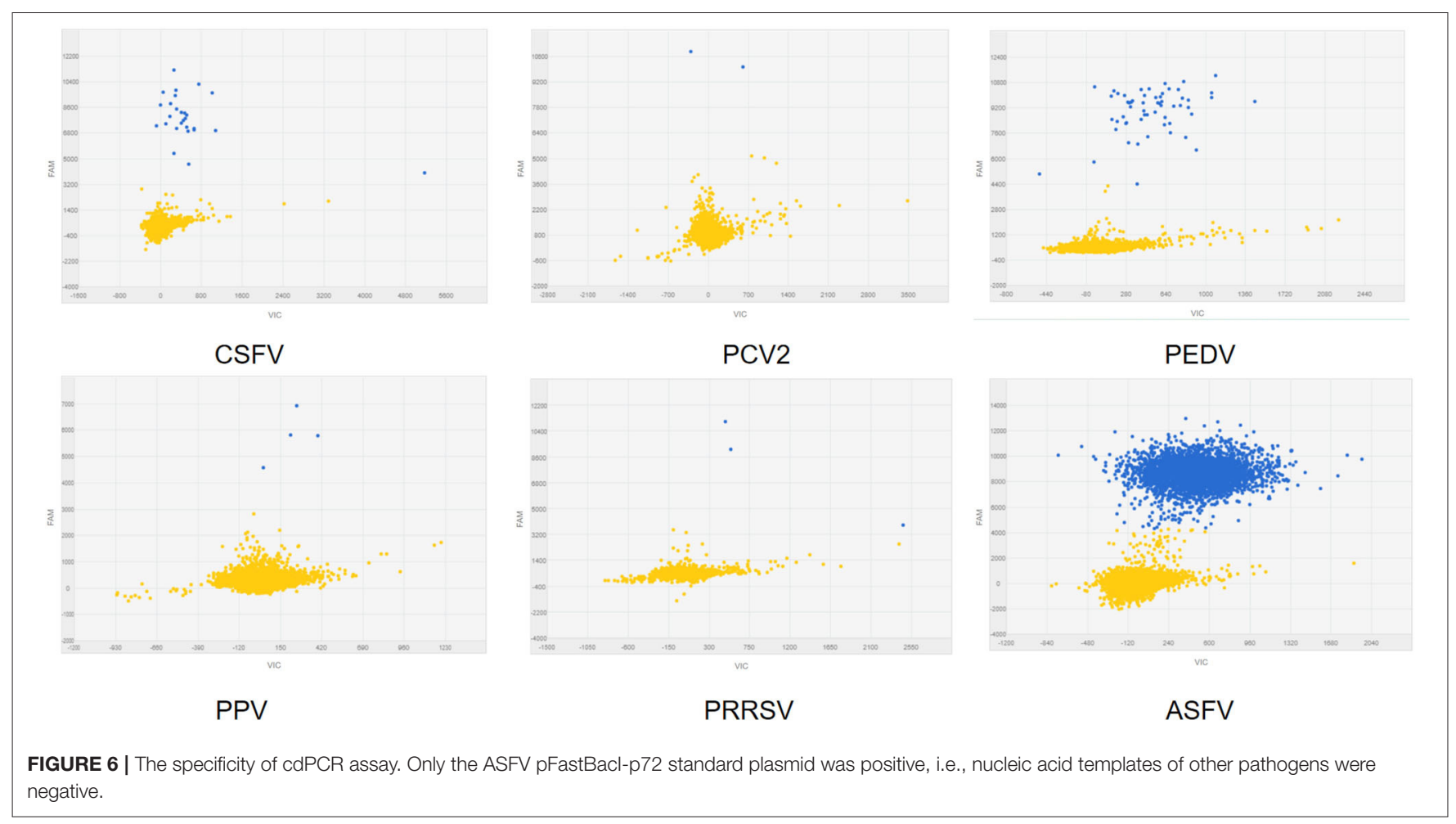

respectively, and the kappa value was $0.800(P<0.0001)$. Those seven samples with inconsistent results between two assays were tested with cdPCR three times to exclude false-positive events, and negative and positive controls were included in all trials. All seven samples were declared as positive samples by cdPCR tests. In addition, the quantitative agreements were evaluated using Pearson correlation and linear regression analysis on 44 positive samples. The quantitative analysis of the correlation between the two showed that they had a good correlation because the $R^{2}$ value of linear regression was $0.864(P<0.0001)$ (Figure 8D). The standard deviation of cdPCR was lower than that of the kit (Figure 8E). Bland and Altman analyses plots (Figure 8F) demonstrated that 1.449\% (1/69) dots were outside the region between $95 \%$ lower limit of agreement and $95 \%$ upper limit of agreement with that of the bias value. Within the consistency limit, the absolute value of the difference between the concentration of the sample to be measured by cdPCR and qPCR was $1,762.59$ copies/ml (the top point in Figure $\mathbf{8 F}$ ), and the average value of the difference was 54.85 copies $/ \mathrm{ml}$ determined by Graphpad Prism 7.04.

Above all, the cdPCR technology developed in this study had comparable performances with the qPCR approved by OIE as well as VetMAX ${ }^{\mathrm{TM}}$ African Swine Fever Virus Detection Kit in terms of detecting ASFV clinical samples.

\section{DISCUSSION}

ASFV has been widely spreading outside Africa to Europe (30, 31) and most recently to Georgia (32), China (33), Cambodia (12), South America $(21,34,35)$, and so on, even to reach
TABLE 2 | Concentration of CSFV, PPV, PCV2, PRRSV, and PEDV, and ASFV standard plasmid by cdPCR assay.

\begin{tabular}{lc}
\hline Isolation virus & Mean concentration $(\mathbf{c o p i e s} / \boldsymbol{\mu l})$ \\
\hline CSFV & 1.027 \\
PPV & 0.616 \\
PRRSV & 0.532 \\
PEDV & 1.391 \\
PCV2 & 0.692 \\
ASFV & 845.11 \\
\hline
\end{tabular}

almost every corner of the world, which is a significant transboundary and emerging virus $(36,37)$. ASF is a serious and highly contagious disease with high mortality, causing acute hemorrhagic fever in domestic pigs and wild boars (3840). Hence, ASF was the biggest threat to the world pork industry (41). Although vaccination is the preferred method for controlling the disease, the development of safe vaccines to protect pigs from ASFV has not achieved significant success since the first isolation of ASFV (42). Because there are no safe and efficacious vaccines, the key of current surveillance and control measures against ASF is firstly to cut off the transmission of the pathogen once ASFV clinical symptoms are observed. Then, diagnosis and confirmation of ASFV require laboratory testing. The traditional method of diagnosing ASFV is using qPCR to measure the ASFV genomic DNA. However, the quality of the standard curve affects the accuracy quantification of qPCR. If the standard curve is unstable, ASFV DNA quantification will 
A

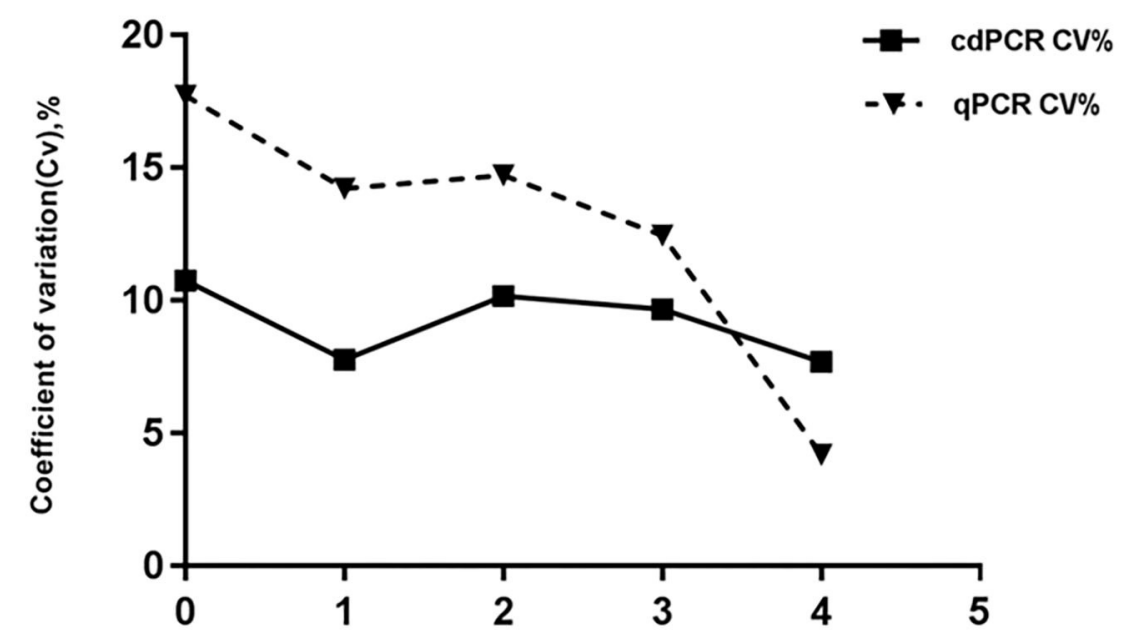

Absolute copies expected (log transformed)

B

\begin{tabular}{|c|c|c|c|}
\hline & cdPCR & qPCR & Difference in CV\% \\
\hline Average CV\% & $9.25 \%$ & $12.67 \%$ & $26.99 \%$ \\
\hline
\end{tabular}

FIGURE 7 | Repeatability analysis. (A) Trend line comparing coefficient of variation (CV\%) for cdPCR and GPCR at different concentration points. The square with the full line stands for $\mathrm{CV} \%$ of cdPCR and the triangle with the dotted line indicated as the CV\% of qPCR. (B) Table shows that average CV\% value of cdPCR (9.25\%) is lower than that of qPCR (12.67\%).

be inexact (43). Additionally, CT values in $\mathrm{qPCR}$ related to amplification efficiencies are obtained from the amplification of standards and the samples. Also, several factors, such as inhibitors, amount of total DNA, and variations between the primers and the probe, may cause the false amplification of the templates, resulting in the CT values going up. Digital PCR as a novel approach to nucleic acid quantification has been used in several aspects with equal or superior performance to qPCR.

Digital PCR can realize an absolute target quantification without standards and the standard curve. Nanofluidic cdPCR running on QuantStudio 3D digital PCR platform (Applied Biosystems) has been applied as a useful tool for sensitive and accurate detection of norovirus low-copy targets (28), quantification of bacterial pathogens (44), quantifying microRNAs in infarction patients (45), and detection of enterotoxigenic Bacteroides fragilis (46). Although droplet digital PCR has been reported being applied to detecting ASFV (47), in this paper, we applied nanofluidic cdPCR on QuantStudio 3D digital PCR platform to diagnose ASF for the first time and assess the applicability of detection ASFV by using cdPCR on aspects such as sensitivity, specificity, reproducibility, among others.

The 53 complete ASFV genome sequences in the GenBank database were aligned, and a suite of primers and an MGB probe were designed based on a highly conserved fragment of the B646L gene coded p72 protein. Various properties of cdPCR assays, such
TABLE 3A | Testing of clinical samples by cdPCR and qPCR assay approved by OIE.

\begin{tabular}{lcccc}
\hline Samples & \multicolumn{3}{c}{ qPCR approved by OIE } & Summary \\
\cline { 2 - 4 } & & Positive & Negative & \\
\hline \multirow{2}{*}{ cdPCR } & Positive & 46 & 4 & 50 \\
& Negative & 2 & 17 & 19 \\
\hline Summary & 48 & 21 & 69 \\
\hline
\end{tabular}

TABLE 3B | Testing of clinical samples by cdPCR and commercial kit.

\begin{tabular}{lcccc}
\hline Samples & \multicolumn{3}{c}{ qPCR (commercial kit) } & Summary \\
\cline { 2 - 4 } & & Positive & Negative & \\
\hline \multirow{2}{*}{ cdPCR } & Positive & 44 & 6 & 50 \\
& Negative & 1 & 18 & 19 \\
\hline Summary & 45 & 24 & 69 \\
\hline
\end{tabular}

as sensitivity, repeatability, and coincidence rate, were evaluated after optimizing reaction conditions. The linearity analysis of cdPCR detection was performed using 10-fold diluted ASFV standard plasmid as templates, with initial concentration of $10^{4}$ 


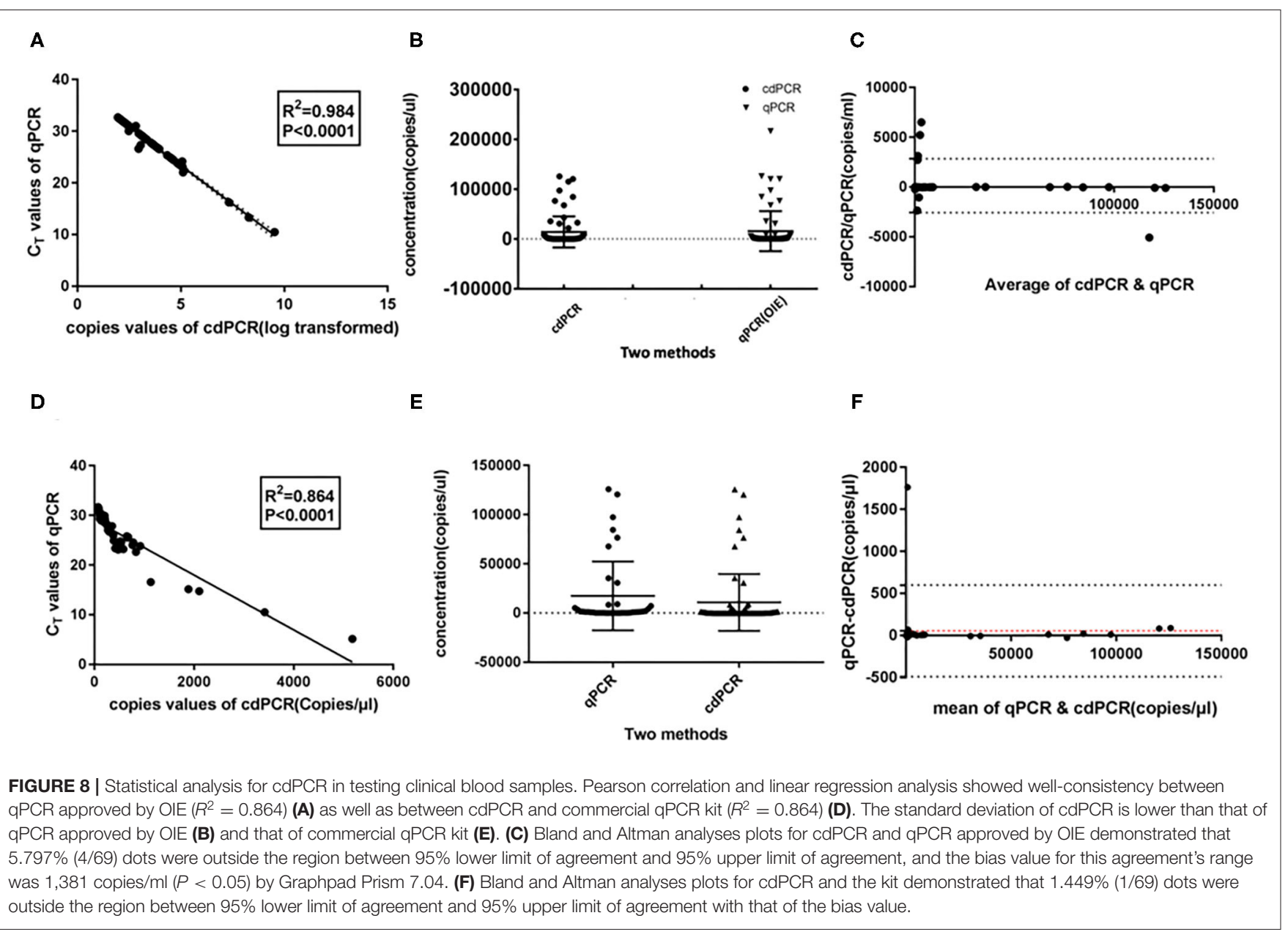

to $10^{-1}$ copies $/ \mathrm{ml}$. The results showed that the limit detection of cdPCR [30.1995 copies per reaction $(n=3)$ ] was approximately 33 times higher than that of qPCR approved by OIE $(1,000$ copies per reaction) (12). Also, the limit detection of cdPCR did correlate well with that of an improved new real-time PCR assay established by Tignon et al. (5.7-57 copies per reaction) (21). The sensitivity of the cdPCR detection method has been significantly improved.

The statistics offer further support in that cdPCR is a perfect tool to detect ASFV. Detecting 69 inactivated clinical serum samples by cdPCR and other techniques showed good consistency with cdPCR and qPCR approved by OIE as well as VetMAX ${ }^{\mathrm{TM}}$ African Swine Fever Virus Detection Kit (Thermofisher, US). The positive detection rate of the cdPCR method established in this study was $72.46 \%$ (50/69), which had a better performance than both qPCR approved by OIE $[69.57 \%$ (48/69)] and VetMAX ${ }^{\mathrm{TM}}$ African Swine Fever Virus Detection Kit $[65.22 \%(45 / 69)]$. Additionally, the cdPCR assay did not react with other swine viruses. Both Bland and Altman analyses and line regression analysis exhibited that cdPCR carried out comparably better than the other two methods.

There are some limitations of the novel cdPCR. That specific equipment is required for nanofluidic cdPCR, which makes it hard to popularize and be widely applied. A specialized nanofluidic chip that accompanies QuantStudio 3D digital PCR platform is a little bit expensive. So, qPCR assay is more economical than cdPCR. Also, cdPCR can only amplify a maximum of 24 samples in a single run, 72 samples fewer than qPCR for a single run. Although this shortcoming of cdPCR can be overcome by adding the number of the ProFlex ${ }^{\mathrm{TM}} 2 \times$ Flat PCR System or Dual Flat Block GeneAmp ${ }^{\text {TM }}$ PCR System (Applied Biosystem, US), the cost is too high. Therefore, qPCR is more applicable in detecting large numbers of clinical samples than cdPCR. However, cdPCR is suitable for the quantification of low copy numbers, especially when the laboratory standard of quantification qPCR for virus genomic DNA/RNA is limited (44). Taken together, the method of using cdPCR to detect ASFV in serum samples has been established and feasible. The cdPCR, as a good tool, can be applied to the absolute quantification of ASFV.

\section{DATA AVAILABILITY STATEMENT}

The raw data supporting the conclusions of this article will be made available by the authors, without undue reservation.

\section{ETHICS STATEMENT}

Ethical statement is not applicable because sample collection has been gathered. Written informed consent was obtained 
from the owners for the participation of their animals in this study.

\section{AUTHOR CONTRIBUTIONS}

AW contributed to study design, laboratory supervision, and manuscript editing. RJ contributed to study design, doing experiments, data analysis and manuscript drafting, editing, and writing. HL contributed significantly to the collection of laboratory data. YC, JZ, YW, and WZ contributed to laboratory quality control and data collection. YL polished the language of the manuscript. PD helped perform the analysis with constructive discussions. GZ and AW contributed to study design, laboratory supervision, and manuscript editing. All authors contributed to the article and approved the submitted version.

\section{REFERENCES}

1. Olesen A, Belsham G, Bruun Rasmussen T, Lohse L, Bødker R, Halasa $\mathrm{T}$, et al. Potential routes for indirect transmission of African swine fever virus into domestic pig herds. Transbound Emerg Dis. (2020) 67:1472-84. doi: $10.1111 /$ tbed 13538

2. Li C, Chai Y, Song H, Weng C, Qi J, Sun Y, et al. Crystal structure of African swine fever virus dUTPase reveals a potential drug target. mBio. (2019) 10:1-11. doi: 10.1128/mBio02483-19

3. He Q, Yu D, Bao M, Korensky G, Chen J, Shin M, et al. High-throughput and all-solution phase African Swine Fever Virus (ASFV) detection using CRISPRCas12a and fluorescence based point-of-care system. Biosens Bioelectron. (2020) 154:112068. doi: 10.1016/j.bios.2020112068

4. Yoon H, Hong S, Lee I, Yoo D, Jung C, Lee E, et al. Clinical symptoms of African swine fever in domestic pig farms in the Republic of Korea, 2019. Transbound Emerg Dis. (2020) 67:2245-8. doi: 10.1111/tbed13552

5. Meng XY, Zhang H, Luo Y, Sun Y, Li Y, Abid M, et al. Cross-priming amplification combined with immunochromatographic strip for rapid onsite detection of African swine fever virus. Sens Actuators B Chem. (2018) 274:304-5. doi: 10.1016/j.snb.2018.07164

6. Galindo I, Alonso C. African swine fever virus: a review. Viruses. (2017) 9:103. doi: 10.3390/v9050103

7. Liu Q, Ma B, Qian N, Zhang F, Tan X, Lei J, et al. Structure of the African swine fever virus major capsid protein p72. Cell Res. (2019) 29:953-5. doi: 10.1038/s41422-019-0232-x

8. Boer CJD, Hess WR, Dardiri AH. African swine fever. Adv Vet Med. (2019) 8:191-4. doi: 10.1016/S0166-3542(98)00015-1

9. Gaudreault NN, Richt JA. Subunit vaccine approaches for African swine fever virus. Vaccines. (2019) 7:56. doi: 10.3390/vaccines70 20056

10. Sánchez EG, Pérez-Núñez D, Revilla Y. Development of vaccines against African swine fever virus. Virus Res. (2019) 265:150-5. doi: 10.1016/j.virusres.2019.03022

11. Revilla Y, Pérez-Núñez D, Richt JA. African swine fever virus biology and vaccine approaches. Adv Virus Res. (2018) 100:41. doi: 10.1016/bs.aivir.2017.10002

12. OIE-WAHID. World Animal Health Information Database (Wahid). World Organisation for Animal Health (OIE). (2017). [Cited World Animal Health Information System (WAHIS)]. Available online at: http://www.Oie.Int/ wahis/public.Php?Page=home (accessed on March 27, 2017).

13. Mehlhorn H. World Organisation for Animal Health (OIE). Berlin Heidelberg: Springer (2015).

14. Agüero M, Fernández J, Romero L, Mascaraque CS, Arias M, SánchezVizcaíno JM. Highly sensitive PCR assay for routine diagnosis of African swine fever virus in clinical samples. J Clin Microbiol. (2003) 41:4431-4. doi: 10.1128/JCM.41.9.4431-44342003

\section{FUNDING}

This study was supported by Zhongyuan high-level talents special support plan (204200510005), the National Key Research and Development Program of China (2016YFD0500704), the National Natural Science Foundation of African Swine Fever Key Project (31941003), the National Natural Science Foundation project (31941001), and the 1125 Talent Gathering Plan project of Zhengzhou.

\section{ACKNOWLEDGMENTS}

We thank Dr. Yilin Bai of Northwest Agriculture and Forestry University in helping us contact the instrument. Also, we thank Ms. Xiaolian Hu, the technician of Themofisher, for the instruction of the experiment.

15. King DP, Reid SM, Hutchings GH, Grierson SS, Wilkinson PJ, Dixon LK, et al. Development of a TaqMan ${ }^{\dagger}$ PCR assay with internal amplification control for the detection of African swine fever virus. J Virol Methods. (2003) 107:9. doi: 10.1016/S0166-0934(02)00189-1

16. Wang A, Jia R, Liu Y, Zhou J, Zhang G. Development of a novel quantitative real-time PCR assay with lyophilized powder reagent to detect African swine fever virus in blood samples of domestic pigs in China. Transbound Emerg Dis. (2019) 67:284-97. doi: 10.1111/tbed13350

17. Shi X, Liu X, Wang Q, Das A, Ma G, Xu L, et al. A multiplex real-time PCR panel assay for simultaneous detection and differentiation of 12 common swine viruses. J Virol Methods. (2016) 236:258-65. doi: 10.1016/j.jviromet.2016.08005

18. Giammarioli M, Pellegrini C, Casciari C, Mia GMD. Development of a novel hot-start multiplex PCR for simultaneous detection of classical swine fever virus, African swine fever virus, porcine circovirus type 2, porcine reproductive and respiratory syndrome virus and porcine parvovirus. Vet Res Commun. (2008) 32:255-62. doi: 10.1007/s11259-007-9026-6

19. Erickson A, Fisher M, Furukawa-Stoffer T, Ambagala A, Hodko D, Pasick J, et al. A multiplex reverse transcription PCR and automated electronic microarray assay for detection and differentiation of seven viruses affecting swine. Transbound Emerg Dis. (2018) 65:e272-83. doi: 10.1111/tbed.12749

20. Fernández-Pinero J, Gallardo C, Elizalde M, Robles A, Gómez C, Bishop $\mathrm{R}$, et al. Molecular diagnosis of African swine fever by a new real-time PCR using universal probe library. Transbound Emerg Dis. (2013) 60:48-58. doi: 10.1111/j.1865-1682.2012.01317x

21. Tignon M, Gallardo C, Iscaro C, Hutet E, Van der Stede Y, Kolbasov D, et al. development and inter-laboratory validation study of an improved new real-time PCR assay with internal control for detection and laboratory diagnosis of African swine fever virus. J Virol Methods. (2011) 178:161-70. doi: 10.1016/j.jviromet.2011.09007

22. Hindson B, Ness K, Masquelier D, Belgrader P, Heredia N, Makarewicz A, et al. High-throughput droplet digital PCR system for absolute quantitation of DNA copy number. Anal Chem. (2011) 83:8604-10. doi: 10.1021/ac202028g

23. Ahmed FE, Ahmed NC, Gouda MM. Quantification of MicroRNAs for the diagnostic screening of colon cancer in human stool by absolute digital(d) PCR. Surg Case Stud Open Access J. (2018) 1:110-8. doi: 10.32474/SCSOAJ.2019.01000125

24. Maynard CL, Wong WKM, Hardikar AA, Joglekar MV. Droplet digital PCR for measuring absolute copies of gene transcripts in human islet-derived progenitor cells. Methods Mol Biol. (2019) 37-48. doi: 10.1007/978-1-4939-9631-5_4

25. Handschuh L, Kazmierczak M, Milewski MC, Goralski M, Luczak M, Wojtaszewska M, et al. Gene expression profiling of acute myeloid leukemia samples from adult patients with AML-M1 and -M2 through boutique microarrays, real-time PCR and droplet digital PCR. Int J Oncol. (2018) 52:656-78. doi: 10.3892/ijo.20174233 
26. Strain MC, Lada SM, Luong T, Rought SE, Gianella S, Terry VH, et al. Highly precise measurement of HIV DNA by droplet digital PCR. PLOS ONE. (2013) 8:e55943. doi: 10.1371/journal.pone0055943

27. Taylor SC, Laperriere G. Germain H. Droplet Digital PCR versus qPCR for gene expression analysis with low abundant targets: from variable nonsense to publication quality data. Sci Rep. (2017) 7:1-8. doi: 10.1038/s41598-017-02217-x

28. Monteiro S, Santos R. Nanofluidic digital PCR for the quantification of Norovirus for water quality assessment. PLoS ONE. (2017) 12:e0179985. doi: 10.1371/journal.pone0179985

29. Burns M, Valdivia H. Modelling the limit of detection in realtime quantitative PCR. Eur Food Res Technol. (2008) 226:1513-24. doi: 10.1007/s00217-007-0683-z

30. Wozniakowski G, Kozak E, Kowalczyk A, Lyjak M, Pomorska-Mol M, Niemczuk K, et al. Current status of African swine fever virus in a population of wild boar in eastern Poland (2014-2015). Arch Virol. (2016) 161:189-95. doi: 10.1007/s00705-015-2650-5

31. Pejsak Z, Truszczyński M, Niemczuk K, Kozak E, Markowska-Daniel I. Epidemiology of African swine fever in Poland since the detection of the first case. Pol J Vet Sci. (2014) 17:665-72. doi: 10.2478/pjvs-2014-0097

32. Sánchez-Cordón P, Montoya M, Reis A, Dixon L. African swine fever: a re-emerging viral disease threatening the global pig industry. Vet J. (2018) 233:41-8. doi: 10.1016/j.tvjl.2017.12025

33. Zhou X, Li N, Luo Y, Liu Y, Miao F, Chen T, et al. Emergence of African swine fever in China, 2018. Transbound Emerg Dis. (2018) 65:1482-4. doi: $10.1111 /$ tbed 12989

34. Wormington JD, Golnar A, Poh KC, Kading RC, Martin E, Hamer SA. Risk of african swine fever virus sylvatic establishment and spillover to domestic swine in the united states. Vector Borne Zoonotic Dis. (2019) 19:506-11. doi: 10.1089/vbz.20182386

35. Golnar AJ, Martin E, Wormington JD, Kading RC, Teel PD, Hamer SA. Reviewing the potential vectors and hosts of african swine fever virus transmission in the united states. Vector Borne Zoonotic Dis. (2019) 7:197. doi: 10.1089/vbz.20182387

36. Karger A, Pérez-Núñez D, Urquiza J, Hinojar P, Alonso C, Freitas F, et al. An update on African swine fever virology. Viruses. (2019) 11:864. doi: 10.3390/v11090864

37. Arabyan E, Kotsynyan A, Hakobyan A, Zakaryan H. Antiviral agents against African swine fever virus. Virus Res. (2019) 270:197669. doi: 10.1016/j.virusres.2019197669

38. Simões M, Freitas F, Leitão A, Martins C, Ferreira F. African swine fever virus replication events and cell nucleus: new insights and perspectives. Virus Res. (2019) 270:197667. doi: 10.1016/j.virusres.2019197667

39. James H, Ebert K, McGonigle R, Reid S, Boonham N, Tomlinson J, et al. Detection of African swine fever virus by loop-mediated isothermal amplification. J Virol Methods. (2010) 164:68-74. doi: 10.1016/j.jviromet.2009.11.034
40. Hübner A, Petersen B, Keil G, Niemann H, Mettenleiter T, Fuchs W. Efficient inhibition of African swine fever virus replication by CRISPR/Cas9 targeting of the viral p30 gene (CP204L). Sci Rep. (2018) 8:1449. doi: 10.1038/s41598-018-19626-1

41. Sánchez-Cordón P, Nunez A, Neimanis A, Wikström-Lassa E, Montoya M, Crooke HD. Gavier-Widén. African swine fever: disease dynamics in wild boar experimentally infected with ASFV isolates belonging to genotype I and II. Viruses. (2019) 11:852. doi: 10.3390/v110 90852

42. Sang H, Miller G, Lokhandwala S, Sangewar N, Waghela S, Bishop R, et al. Progress toward development of effective and safe African swine fever virus vaccines. Front Vet Sci. (2020) 7:84. doi: 10.3389/fvets.2020 00084

43. Busby E, Whale AS, Ferns RB, Grant PR, Garson JA. Instability of 8E5 calibration standard revealed by digital PCR risks inaccurate quantification of HIV DNA in clinical samples by qPCR. Sci Rep. (2017) 7:1209. doi: 10.1038/s41598-017-01221-5

44. Ricchi M, Bertasio C, Boniotti MB, Vicari N, Russo S, Tilola M, et al. Comparison among the quantification of bacterial pathogens by qPCR, dPCR, and cultural methods. Front Microbiol. (2017) 8:1174. doi: 10.3389/fmicb.2017.01174

45. Robinson S, Follo M, Haenel D, Mauler M, Stallmann D, Tewari M. Chipbased digital pcr as a novel detection method for quantifying micrornas in acute myocardial infarction patients. Acta Pharmacol Sin. (2017) 257:247-54. doi: 10.1038/aps.2017136

46. Purcell RV, Pearson J, Frizelle FA, Keenan JI. Comparison of standard, quantitative and digital PCR in the detection of enterotoxigenic Bacteroides fragilis. Sci Rep. (2016) 6:34554. doi: 10.1038/srep34554

47. Wu X, Xiao L, Lin H, Chen S, Yang M, An W, et al. Development and application of a droplet digital polymerase chain reaction (ddPCR) for detection and investigation of African swine fever virus. Can J Vet Res. (2018) 82:70-74. doi: 10.1016/j.meegid.2017.10019

Conflict of Interest: HL, YC, JZ, YL, YW, and WZ were employed by Henan Zhongze Biological Engineering Co. Ltd.

The remaining authors declare that the research was conducted in the absence of any commercial or financial relationships that could be constructed as a potential conflict of interest.

Copyright (C) 2021 Jia, Zhang, Liu, Chen, Zhou, Liu, Ding, Wang, Zang and Wang. This is an open-access article distributed under the terms of the Creative Commons Attribution License (CC BY). The use, distribution or reproduction in other forums is permitted, provided the original author(s) and the copyright owner(s) are credited and that the original publication in this journal is cited, in accordance with accepted academic practice. No use, distribution or reproduction is permitted which does not comply with these terms. 